\title{
REMARKS ON FLUORESCENCE CONCENTRATION QUENCHING OF RHODAMINE 6G IN METHANOL*†
}

\author{
C. Bojarski, R. Bujko \\ Institute of Physics, Technical University of Gdańsk, Majakowskiego 11/12, \\ 80-952 Gdańsk, Poland \\ AND P. BOJARSKI \\ Institut of Experimental Physics, University of Gdańsk, Gdańsk, Poland
}

(Received June 21, 1990)

It has been shown that the concentration changes in the quantum yield $\eta$ and decay time $\tau$ of fluorescence of rhodamine $6 \mathrm{G}$ in methanol can be explained quantitatively by non-radiative excitation energy transport (NET) from monomers to quenching centres considered as perfect traps. The good agreement of the experimental data with the theoretical curves for the critical radii $R_{\mathrm{OMM}}=55.4 \AA$ and $R_{\mathrm{OMT}}=51.8 \AA$ determined spectroscopically has been obtained. The excitation energy migration and the concentration dependence on the orientation factor occurring in the Förster rate for the NET have been taken into account. The nature of the quenching centres and the effect of material diffusion of active molecules on $\eta$ and $\tau$ have been discussed.

PACS numbers: 33.50.-j, 78.55.Bg

\section{Introduction}

Alcohol solutions of rhodamine $6 \mathrm{G}(\mathrm{Rh} 6 \mathrm{G})$ are often used as active media in dye lasers. It is assumed that in concentrated dye solutions, apart from luminescent molecules (LM), there can exist different types of quenching centres such as dimers,

*The research was sponsored by the Polish Ministry of National Education under Project CPBP 01.06.

tDedicated to Professor Michael Kasha on the occasion of his 70th birthday. 
oligomers, excimers as well as statistical pairs [1-9]. These centres can absorb the exciting light or constitute traps for the excitation energy transferred to them from the excited monomers.

The investigations of the fluorescence concentration quenching of rhodamine $6 \mathrm{G}$ in solutions have been reported in many papers [9-19]. Recently quantum yield $\eta$ and fluorescence decay time $\tau$ measurements of rhodamine $6 \mathrm{G}$ in methanol in a very wide concentration range have been carried out [5, 20,21]. Also absorption and fluorescence spectra of monomers and quenching centres have been there investigated. The observed concentration changes of $\eta$ and $\tau$ were explained in terms of non-radiative energy transfer from monomers $M$ to statistical pairs (traps) $T$, as well as material diffusion of molecules $\mathrm{M}$ and $\mathrm{T}$.

It should be emphasized that the formulae applied in [5] for the description of the changes in $\eta$ and $\tau$ as a function of concentration do not result from a compact theory, having an approximate character since they do not take into account the excitation energy migration.

In the present paper it will be shown that the experimental results mentioned above can be described and interpreted within the framework of a coherent statistical theory which takes into account the energy migration (EM), assuming that quenching centres play the role of perfect traps. In what follows we shall discuss the nature of the quenching centres and the effect of material diffusion of $M$ and T molecules upon $\eta$ and $\tau$.

\section{Quantitative analysis}

Concentration changes in the quantum yield and the decay time of fluorescence conditioned by long-range NET from monomers $M$ to traps $T$ can be described by the expressions [22-24]:

$$
\begin{gathered}
\eta=\eta_{\mathrm{OM}}(1-f) /(1-\alpha f) \\
\tau=\tau_{\mathrm{OM}} \frac{1+\gamma^{2}-\left(1.5+\gamma^{2}\right) f+\alpha f\left(f+\gamma^{2}-0.5\right)-\alpha \gamma^{2}}{(1-f)(1-\alpha f)}
\end{gathered}
$$

where

$$
f \equiv f(\gamma)=\pi^{1 / 2} \gamma \exp \left(\gamma^{2}\right)[1-\operatorname{erf}(\gamma)]
$$

and

$$
\begin{gathered}
\gamma=\gamma_{\mathrm{M}} / 2^{1 / 2}+\gamma_{\mathrm{T}}=\pi^{1 / 2}\left[2^{-1 / 2} C_{\mathrm{M}} / C_{\mathrm{OMM}}+C_{\mathrm{T}} / C_{\mathrm{OMT}}\right] / 2, \\
\alpha=2^{-1 / 2} \gamma_{\mathrm{M}} / \gamma, \\
\gamma=\gamma_{\mathrm{M}} / 2^{1 / 2}+\gamma_{\mathrm{T}}, \\
\gamma_{\mathrm{M}}=\left(\pi^{1 / 2} / 2\right) C_{\mathrm{M}} / C_{\mathrm{OMM}}, \\
\gamma_{\mathrm{T}}=\left(\pi^{1 / 2} / 2\right) C_{\mathrm{T}} / C_{\mathrm{OMT}} .
\end{gathered}
$$

$\eta_{\mathrm{OM}}$ and $\tau_{\mathrm{OM}}$ denote the quantum yield and the mean decay time of fluorescence when $C_{\mathrm{T}}=0$, whereas $C_{\mathrm{M}}, C_{\mathrm{T}}, C_{\mathrm{OMM}}, C_{\mathrm{OMT}}$ denote concentrations and critical concentrations of monomers $M$ and traps $T$, respectively. 
Formulae (1) and (2) were obtained assuming the molecules $M$ and $T$ to be randomly distributed in an inactive and viscous medium. In these formulae, the correlations between the configurations of the $\mathrm{M}$ and $\mathrm{T}$ molecules in the vicinity of successively excited monomers, as well as the EM process in the set of monomers have been taken into account $[25,26]$.

In order to compare the experimental values of $\eta$ and $\tau$ with those obtained from expressions (1) and (2), the values of concentrations $C_{\mathrm{M}}$ and $C_{\mathrm{T}}$ as well as critical concentrations $C_{\mathrm{OMM}}$ and $C_{\mathrm{OMT}}$ of monomers and traps, respectively, should be known. Let us now consider two cases, when traps are formed by:

(a) ground state dimers and (b) statistical pairs.

Concentrations $C_{\mathrm{M}}=x_{\mathrm{M}} C$ and $C_{\mathrm{T}}=x_{\mathrm{T}} C / 2=\left(1-x_{\mathrm{M}}\right) C / 2$ have been determined from expressions:

$$
\begin{array}{ll}
x_{\mathrm{M}}=(\sqrt{1+4 K C}-1) / 4 K C & \text { in case (a) } \\
\text { and } \quad x_{\mathrm{M}}=\exp \left(-V_{\mathrm{T}} N_{\mathrm{A}} C\right) & \text { in case (b), }
\end{array}
$$

where $C=C_{\mathrm{M}}+2 C_{\mathrm{T}}, K=C_{\mathrm{T}} / C_{\mathrm{M}}^{2}$ is the dimerization constant, $V_{\mathrm{T}}=(4 / 3) \pi R_{\mathrm{T}}^{3}$, $R_{\mathrm{T}}$ - the distance between Rh6G molecules in a pair, $N_{\mathrm{A}}$ - the Avogadro constant. From the spectroscopic measurements $C_{\text {омM }}=2.33 \times 10^{-3} \mathrm{~mol} / \mathrm{l}$ was obtained [27]. Critical concentration $C_{\mathrm{OMT}}=2.87 \times 10^{-3} \mathrm{~mol} / \mathrm{l}$ was determined from the monomer fluorescence spectrum and the trap absorption spectrum, taken from Ref. [21] for the value of orientation factor $\overline{\kappa^{2}}=2 / 3$.

When calculating the reduced concentrations $\gamma$, we have taken into consideration the variation in $\overline{\kappa^{2}}$ [see. Eq. (10)], i.e. also the change in the values of $C_{\mathrm{OMM}}$ and $C_{\text {OMT }}$ with $C$. Figure 1 illustrates the comparison of the experimental values of $\eta$ and $\tau$ and taken from Ref. [5] with those obtained from Eqs. (1) and (2). Solid and dashed curves, $\eta$ and $\tau$, correspond to cases (a) and (b), respectively. These curves were plotted for the same values of $C_{\mathrm{OMM}}$ and $C_{\mathrm{OMT}}$. They only differ in the values of $C_{\mathrm{M}}$ and $C_{\mathrm{T}}$, determined from (5) and (6). Values of $K$ and $R_{\mathrm{T}}$ are the best fit parameters. Although $C_{\mathrm{T}}$ and $C_{\mathrm{M}}$ calculated for pairs and dimers differ considerably in the range of the highest $C$ (Fig. 2), the solid and dashed curves, $\eta$ and $\tau$, coincide almost in the whole concentration range. Therefore, the traps cannot be in this way identified as the ground state dimers or pairs. However, in contrary to statistical pairs, appearing of dimers has been well experimentally documented [2, 28] and has clear physical interpretation [29]. It should be noted that the experimental data are in good accordance with those obtained from (1) and (2) for critical distances $R_{\mathrm{OMM}}=55.4 \AA$ and $R_{\mathrm{OMT}}=51.8 \AA$, determined from independent measurements. The value of $R_{\mathrm{OMT}}=44.5 \AA$, determined in [21] as the parameter of the best fit differs significantly from the value of $R_{\text {OMT }}$ given above. It can be noted that the two experimental points of $\eta$ corresponding to the highest concentrations deviate distinctly from the theoretical curves (see Fig. 1). These deviations can be due to the imperfections of the traps $\left(\eta_{\mathrm{OT}}>0\right)$. Also the interactions between higher multipoles in the concentration range considered participate to some extent in the process of NET. Let us add that the translational diffusion of molecules $\mathrm{M}$ and $\mathrm{T}$ in (1) and (2) has totally been neglected. This problem will be discussed in the next chapter. 

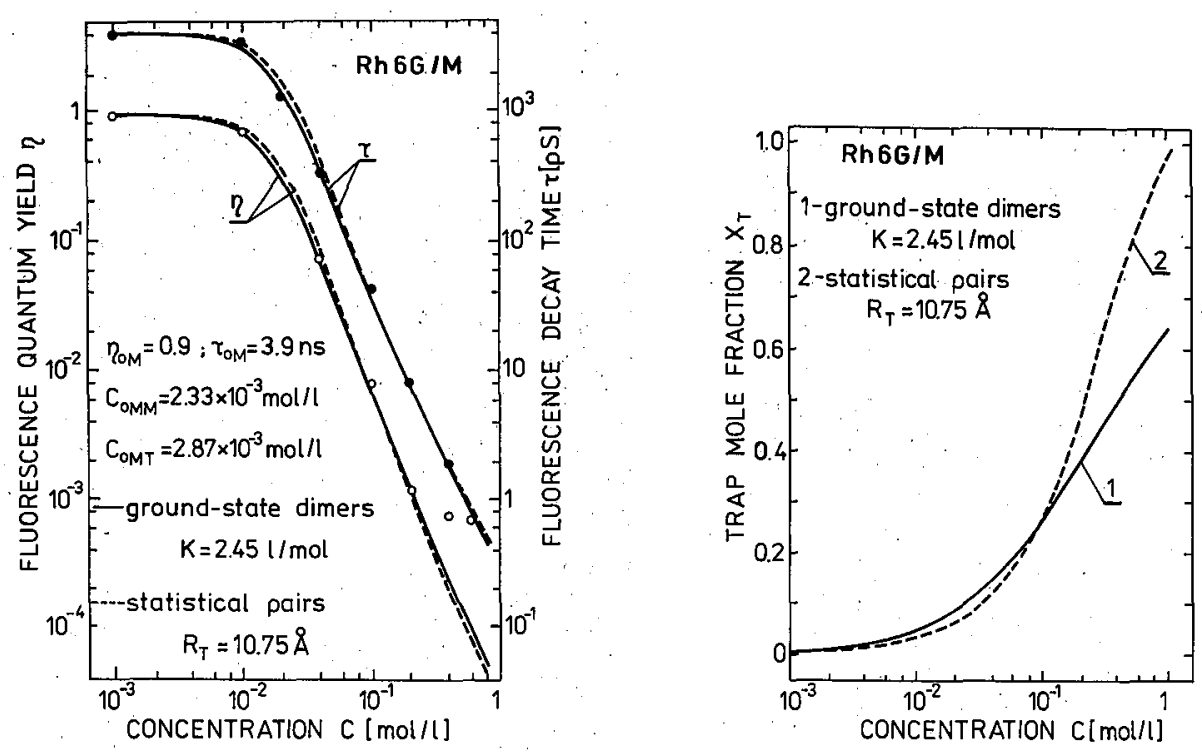

Fig. 1. The dependence of fluorescence decay time $\tau$ and quantum yield $\eta$ on the concentration of rhodamine $6 \mathrm{G}$ in methanol: $0, \bullet-$ experimental data taken from Ref. [5], curves $\eta(C), \tau(C)$ have been calculated using (1) and (2), respectively.

Fig. 2. Mole fraction of traps vs. concentration: solid curve - calculated from (5), dashed curve - calculated from (6).

\section{Discussion and final remarks}

In the case of systems of low viscosity such as that considered herein, where the average translation of the luminescent molecules during the lifetime in the excited state:

$$
\bar{r}_{0} \equiv\left[\bar{r}^{2}\right]^{1 / 2}=\left[2 D \tau_{\mathrm{OP}}\right]^{1 / 2}
$$

is comparable to the critical distance $R_{\mathrm{OMX}}(\mathrm{X}=\mathrm{M}, \mathrm{T}$ ), one may expect that the diffusion of LM will enhance the effectiveness of the NET process [30]. In expression (7), $D=D_{\mathrm{M}}+D_{\mathrm{T}}$, where $D_{\mathrm{M}}$ and $D_{\mathrm{T}}$ are the diffusion coefficients of molecules $\mathrm{M}$ and $\mathrm{T}$, respectively. For rhodamine $6 \mathrm{G}$ in methanol at room temperature, $D_{\mathrm{M}} \approx$ $D_{\mathrm{T}}=5.97 \times 10^{-6} \mathrm{~cm}^{2} / \mathrm{s}[27]$ and $\tau_{\mathrm{OM}}=3.9 \mathrm{~ns}[5]$, hence $\vec{r}_{0}=30.52 \AA$, while $R_{\text {OMM }}=55.4 \AA$ and $R_{\text {OMT }}=51.8 \AA$. Therefore, the effect of diffusion on the course of $\eta(c)$ and $\tau(c)$ should be expected. However, in the range of high concentrations $C_{\mathrm{M}}$ and $C_{\mathrm{T}}$, the time of localization of the excitation energy at molecule $\mathrm{M}^{*}[31$, 32]:

$$
\tau_{1}=\tau_{\mathrm{OM}}\left(\tau / \tau_{\mathrm{OM}}\right)(1-\alpha f)
$$

can be much shorter than $\tau_{\mathrm{OM}}$, and the true distance of diffusion [33]:

$$
\bar{r}=\left(2 D \tau_{1}\right)^{1 / 2}=\bar{r}_{0}\left[\left(\tau / \tau_{\mathrm{OM}}\right)(1-\alpha f)\right]^{1 / 2}=\bar{r}_{0}[\varphi(\gamma)]^{1 / 2}
$$


can be much shorter than $\bar{r}_{0} ; \tau, f, \alpha$ are described by (2), (3) and (4), respectively.
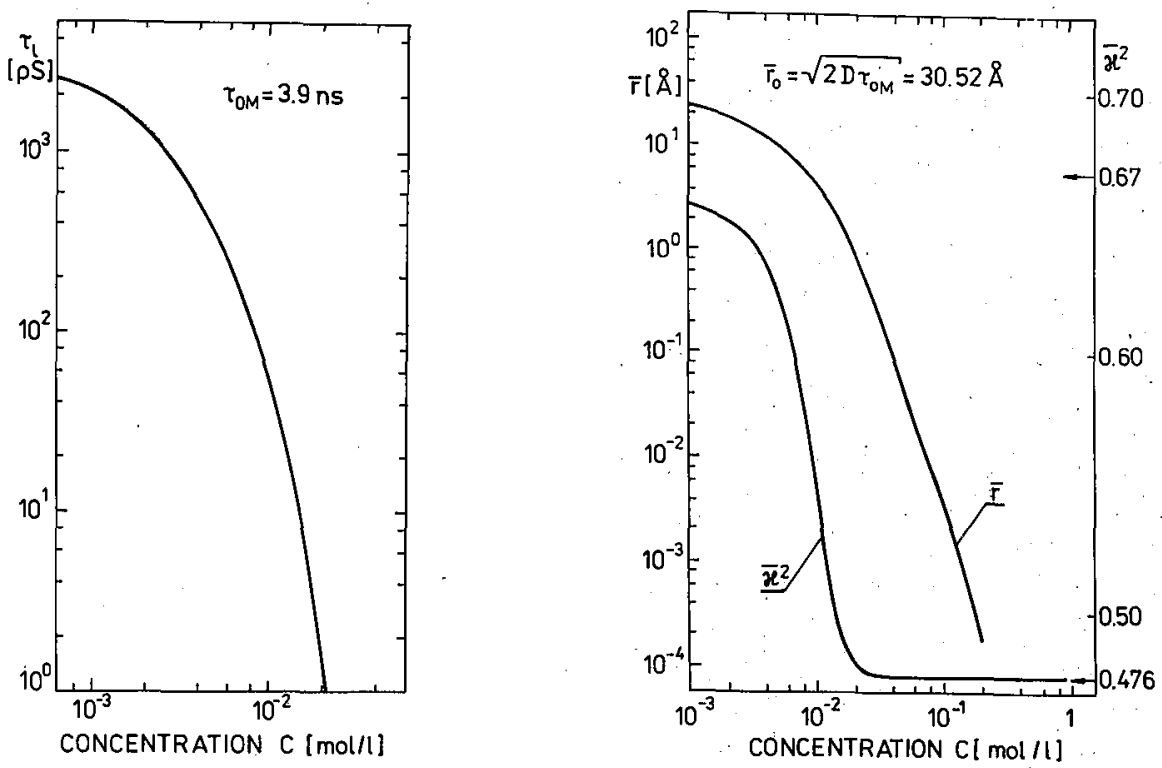

Fig. 3. Mean lifetime of Rh6G molecules $\pi_{l}$ in the excited state vs. dye concentration calculated using Eq. (8) for the same values of parameters as in Fig. 1.

Fig. 4. Actual diffusion length $\bar{r}$ and orientation factor $\overline{\kappa^{2}}$ vs. concentration of rhodamine 6G in methanol; curves $\bar{r}$ and $\overline{\kappa^{2}}$ had been calculated from (9) and (10), respectively.

Figure 3 shows mean lifetime $\tau_{l}$ of Rh6G in methanol as a function of concentration. Already at $C=2 \times 10^{-2} \mathrm{~mol} / \mathrm{l}, \tau_{l}$ reaches a value of a few picoseconds. In such a case, the corresponding value of $\bar{r}$ does not exceed $1 \AA$ (see Fig. 4). One may therefore conclude that in the range of high concentrations, $\left(C>10^{-2} \mathrm{~mol} / \mathrm{l}\right)$ the system of very low viscosity investigated exhibits, for the NET processes considered, the features of the rigid system in which the translational diffusion of LM can be neglected.

The increase in $C$ can induce a change in $\tau_{l}$ from $\pi_{l} \gg \tau_{r}$ to $\tau_{l} \ll \tau_{r}$, thus leading to the decrease in $\overline{\kappa^{2}}$ from $2 / 3$ (fast rotating dipoles [1]) to 0.476 (statistical distribution of immobile dipoles [34]). The values of $\overline{\kappa^{2}}(\gamma)$ from this interval can be calculated using the following formula [31]:

$$
\overline{\kappa^{2}}(\gamma)=(2 / 3) \frac{\varphi(\gamma)}{\varphi(\gamma)+\tau_{r} / \tau_{\mathrm{OM}}}+0.476 \frac{\tau_{r} / \tau_{\mathrm{OM}}}{\varphi(\gamma)+\tau_{r} / \tau_{\mathrm{OM}}}
$$

where $\varphi(\gamma)$ is defined in (9) and $\tau_{r}$ is the rotational relaxation time. For Rh6G molecules in methanol $\tau_{r}=0.11 \mathrm{~ns}$ [27]. Figure 4 shows the concentration changes in $\overline{\kappa^{2}}$ for Rh6G in methanol. Already for $C=2 \times 10^{-2} \mathrm{~mol} / 1, \overline{\kappa^{2}}$ is close to a limiting value of 0.476 . The change of the angular factor value leads to the change in critical concentration. 


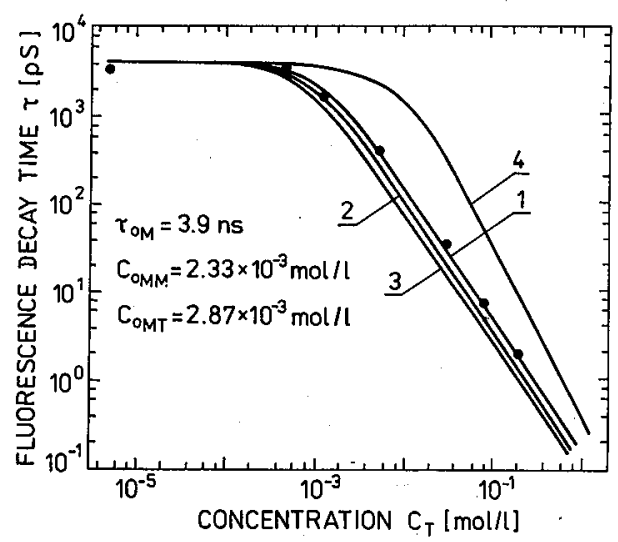

Fig. 5. The dependence of fluorescence decay time on trap concentration for rhodamine 6G in methanol: - - experimental points taken from Ref.[5], theoretical curves calculated from (2); 1 - energy migration (EM), correlations and changes of $\overline{\kappa^{2}(C)}$ taken into account; 2 - correlations neglected; 3 - correlations and changes of $\overline{\kappa^{2}}$ neglected; 4 - Förster-Galanin curve - correlations, changes of $\overline{\kappa^{2}}$ and EM neglected.

Figure 5 shows the fluorescence decay time $\tau$ versus the dimer concentration $C_{\mathrm{T}}$. Curve 1 corresponds to the solid curve $\tau$ in Fig. 1. For comparison, the concentration changes of $\tau$ were calculated from (2) for the same values of parameters $K, C_{\mathrm{OMM}}$ and $C_{\mathrm{OMT}}$ but for $\gamma$ replaced with $\gamma^{\prime}=\gamma_{\mathrm{M}}+\gamma_{\mathrm{T}}$ and $\alpha^{\prime}=\gamma_{\mathrm{M}} / \gamma^{\prime}$ (curve 2 ). In this case, the correlations were neglected. Curve 3 corresponds to the case when the correlations, as well as the concentration changes of the orientation factor were neglected (COMM and $C_{\mathrm{OMT}}$ were calculated for $\overline{\kappa^{2}}=2 / 3$ ). Curve 4 is the Förster-Galanin [35] curve calculated from (2) and (4) for $\alpha=0$ (energy migration also being neglected). That latter curve corresponds to the simplest model of the fluorescence quenching with NET from $\mathrm{M}^{*}$ to $\mathrm{T}$ occurring in a single step only (the model of static quenching).

As is readily seen in Fig. 5, an essential improvement related to this model has been introduced by the energy migration (c.f. curves 4 and 3). Taking into account the correlations and the decrease in the orientation factor results in further corrections (curves 1 and 2) which, in the case of the system considered are comparable and small as compared to those resulting from the energy migration. The dependences analogous to those shown in Fig. 5, can also be observed for the concentration changes in the quantum yield.

We do hope, that the given above analysis of fluorescence concentration quenching of rhodamine $6 \mathrm{G}$ in methanol can be useful in explaining the fluorescence quenching mechanism in other liquid systems in which very strong energy migration and its trapping takes place. 


\section{References}

[1] Th. Förster, Fluoreszenz Organischer Verbindungen, Vandenhoeck und Ruprecht, Göttingen 1951.

[2] V.L. Levshin, Izv. Akad. Nauk SSSR Ser. Fiz. 27, 540 (1963).

[3] G. Obermueller, C. Bojarski, Acta Phys. Pol. A52, 431 (1977).

[4] G.S. Beddard, G. Porter, Nature 260, 366 (1976).

[5] A. Penzkofer, Y. Lu, Chem Phys. 103, 399 (1986).

[6] J. Knoester, J.E. Van Himbergen, J. Chem Phys. 84, 2990 (1986); ibid. 86, 4438 (1987); 86, 3571 (1987).

[7] F. Lopez Arbeloa, I. Llona Gonzalez, P. Ruiz Ojeda, I. Lopez Arbeloa, J. Chem. Soc. Faraday Trans. II 78, 989 (1982).

[8] P. Ruiz Ojeda, I.A. Katima Amashta, J.R. Ochoa, I. Lopez Arbeloa, J. Chem. Soc. Faraday Trans. II 84, 1 (1988).

[9] K.A. Selanger, J. Falnes, T. Sikkeland, J. Phys. Chem. 81, 1960 (1977).

[10] E.G. Baranova, Opt. Spektrosk. 13, 452 (1962).

[11] H.J. Cirkel, L. Ringwelski, F.P. Schäfer, Z. Phys. Chem. Neue Folge 81, 158 (1972).

[12] R.R. Alfano, S.L. Shapiro, N. Yu, Opt. Commun. 7, 191 (1973).

[13] C. Bojarski, G. Obermueller, Acla Phys. Pol. A50, 389 (1976).

[14] K. Szücs, B. Rácz, B. Német, L. Kozma, I. Santa, M. Hilbert, Acta Univ. Szeged. Acta Phys. Chem. 24, 437 (1978).

[15] C. Bojarski, E. Grabowska, Acta Phys. Pol. A60, 397 (1981).

[16] D.R. Lutz, K.A. Nelson, C.K. Gochanour, M.D. Fayer, Chem. Phys. 58,325 (1981).

[17] C. Bojarski, A. Bujko, R. Bujko, Acta Phys. Hung. 59, 307 (1986).

[18] F. Lopez Arbeloa, P. Ruiz Ojeda, I. Lopez Arbeloa, J. Photochem. Photobiol., A: Chemistry 45, 313 (1988).

[19] F. Lopez Arbeloa, P. Ruiz Ojeda, I. Lopez Arbeloa, J. Chem. Soc. Faraday Trans. II 84, 1903 (1988).

[20] Y. Lu, A. Penzkofer, Chem Phys. 107, 175 (1986).

[21] A. Penzkofer, W. Leupacher, J. Lumin. 37, 61 (1987).

[22] C. Bojarski, J. Domsta, Acla Phys. Hung. 30, 145 (1971).

[23] R. Twardowski, C. Bojarski, Z. Naturforsch. Teil A 35, 345 (1980).

[24] A.I. Burshtein, J. Lumin. 34, 201 (1985).

[25] A. Kawski, Photochem. Photobiol. 38, 487 (1983).

[26] C. Bojarski, K. Sienicki, in Focus of Photochemistry and Photophysics, ed. J.F. Rabek, CRC Press, Boca Raton 1989.

[27] C. Bojarski, A. Bujko, R. Bujko, H. Cherek, Acta Phys. Hung. 59, 307 (1986).

[28] Th. Förster, E. König, Z. Elektrochem.; Ber. Bunsenges. Phys. Chem. 61, 344 (1957). 
[29] M. Kasha, H.R. Rawls, A. El-Bayoumi, Pure Appl. Chem. 11, 371 (1965).

[30] V.L. Ermolaev, I.N. Bodunov, J.B. Sveshnikova, T.A. Shahverdov, Bezyzluchatelnyi Perenos Elektronnoy Energii Vozbuzhdeniya, Izd. Nauká, Leningrad 1977.

[31] C. Bojarski, J. Dudkiewicz, Chem. Phys. Lett. 67, 450 (1979).

[32] C. Bojarski, E. Grabowska, Z. Nalurforsch. Teil A 35, 1030 (1980); erratum 35, 1431 (1980).

[33] C. Bojarski, Z. Naturforsch. Teil A 36, 78 (1981).

[34] M.Z. Maksimov, I.M. Rozman, Opt. Spektrosk. 12, 606 (1962).

[35] M.D. Galanin, Trudy Fiz. Inst. Ak. Nauk SSSR 12, 3 (1960). 